\title{
The Neuroimmune Pharmacology of SARS-CoV-2
}

\author{
Santosh Kumar ${ }^{1}$ (]) Sowmya Yelamanchili ${ }^{2}$. Pankaj Seth ${ }^{3}$. Jean M. Bidlack ${ }^{4}$. Gurudutt Pendyala ${ }^{2}$. \\ Sanjay Maggirwar ${ }^{5}$. Sulie L. Chang ${ }^{6}$
}

Received: 15 November 2021 / Accepted: 23 November 2021 / Published online: 22 December 2021

(c) The Author(s), under exclusive licence to Springer Science+Business Media, LLC, part of Springer Nature 2021

\begin{abstract}
This guest commentary introduces "The Neuroimmune Pharmacology of SARS-CoV-2," a special theme issue for The Journal of Neuroimmune Pharmacology led by the Society on NeuroImmune Pharmacology. The issue builds on the Society's Virtual Workshop on COVID-19 held April 9, 2021.
\end{abstract}

Keywords COVID-19 $\cdot$ SARS-CoV-2 Oral history $\cdot$ Diversity $\cdot$ Equity and inclusion $\cdot$ Early-career investigators $\cdot$ Wellbeing $\cdot$ Neuroimmune pharmacology $\cdot$ Therapeutics $\cdot$ Vaccines

This guest commentary briefly describes the papers that are published in the special issue, "The Neuroimmune Pharmacology of SARS-CoV-2." The theme issue materialized as a result of a workshop on COVID-19 that the Society on Neuroimmune Pharmacology (SNIP) organized, with the help of the University of Nebraska Medical Center (UNMC), on April 9, 2021. The workshop covered a number of COVID-19-related research topics, including molecular approaches to COVID-19 pathogenesis and underlying mechanisms, therapeutic and vaccine approaches to

Santosh Kumar

ksantosh@uthsc.edu

Sulie L. Chang

sulie.chang@shu.edu

1 Department of Pharmaceutical Sciences, College of Pharmacy, University of Tennessee Health Science Center, Memphis, TN, USA

2 Department of Anesthesiology, College of Medicine, University of Nebraska Medical Center, Omaha, NE, USA

3 Cellular and Molecular Neuroscience, National Brain Research Center, Panesar, Haryana, India

4 Department of Pharmacology and Physiology, School of Medicine and Dentistry, University of Rochester Medical Center, Rochester, NY, USA

5 Department of Microbiology, Immunology \& Tropical Medicine, George Washington University School of Medicine and Health Sciences, Washington D.C., USA

6 Institute of Neuroimmune Pharmacology and Department of Biological Sciences, Seton Hall University, South Orange, NJ, USA
COVID-19, COVID-19-induced neurological complications, well-being of researchers during the pandemic, and other related topics. Upon completion of the workshop, several preSsenters and non-presenters in the field of neuroimmune pharmacology were invited to write and submit original papers. Altogether, this special issue collects eight articles: this guest commentary, one perspective, two brief reports, one review, and three original research articles.

In this editorial commentary by guest editors introducing the theme issue, we introduce and summarize the invited manuscripts in order of their presentation in the issue's table of contents. First, we present, "The COVID-19 pandemic: Reflections of science, person, and challenge in academic research settings," which collects various perspectives from the Society's spring 2021 virtual workshop symposium focused on well-being and reflections (Bidlack et al. 2021). Then, there are two brief reports: "Cannabis/Cannabinoids for Treating COVID-19 Associated Neuropsychiatric Complications" (Khalsa et al. 2021) and "SARS-CoV-2 spike protein disrupts blood-brain barrier integrity via RhoA activation" (DeOre et al. 2021). Next comes the issue's review paper, "Racial Health Disparity and COVID-19" (Kumar et al. 2021b) followed by three original research articles. One of these primary research papers examines a potential treatment approach using the herbal product, "Eugenol, a component of holy basil (Tulsi) and common spice clove, and reporting that this herbal product inhibits the interaction between SARS-CoV-2 spike S1 and ACE2 to induce therapeutic responses" (Paidi et al. 2021). The next paper describes original meta-analysis research, "Network meta-analysis on 
the changes of amyloid precursor protein expression following SARS-CoV-2 infection" (Camacho et al. 2021). The final manuscript of the theme issue is "Mitochondrial dynamics in SARS-COV2 spike protein treated human microglia: implications for neuro-COVID" (Clough et al. 2021).

\section{Perspective}

\section{SNIP Workshop on the COVID-19 Pandemic}

In collaboration with UNMC, SNIP organized a workshop on COVID-19. Presentation topics ranged from the impact of COVID-19 on health disparity, drug abuse, infectious diseases, and neurological complications, as well as potential therapeutic and vaccine approaches to COVID-19. In advance of this theme issue, the organizing committee of the workshop compiled a brief report (Kumar et al. 2021a) from the four symposia of the workshop: 1) Molecular approaches to COVID-19 pathogenesis and underlying mechanism; 2) Therapeutic and vaccine approaches to COVID-19; 3) Early Career Investigator talks; and 4) Diversity and Inclusion SNIP Committee (DISC) program: Well-being and reflections. The virtual workshop also noted four special talks by NIH officials on COVID-19 and funding opportunities from the National Institute on Alcohol Abuse and Alcoholism (NIAAA) and National Institute on Drug Abuse (NIDA).

Further building on the fourth and last symposium of the virtual workshop, "Well-being and Reflections," edited transcription of a presentation and panel discussion were compiled into an oral history contribution from the field of neuroimmune pharmacology (Bidlack et al. 2021). This perspective shows the impact of the pandemic on the lives of basic science researchers, educators, and students. The symposium began with a talk by Dr. Jeymohan Joseph from the National Institute of Mental Health (NIMD) on, "Neurological and Psychiatric complications of SARS-CoV-2 infection followed by a scientific presentation on targeting innate immunity for the treatment of COVID-19 by a renowned scientist from the St. Jude Children Hospital, Dr. Thirumala-Devi Kanneganti. However, transcriptions provided the bulk of the article's text. The first transcription was derived from a talk by Dr. Santosh Kumar, "Intervention and improved well-being of basic science researchers during the COVID-19 era: a case study." In his presentation, Dr. Kumar's group discussed an intervention study among his researchers to mitigate COVID-19-induced stress without compromising research productivity. Next, the perspective transcribed the discussion from a "Reflection and sharing" panel organized by Dr. Sowmya Yelamanchili, featuring Drs. Jean M. Bidlack, Sylvia Fitting,
Santhi Gorantla, Maria Cecilia G. Marcondes, Loyda M. Melendez, and Ilker K. Sariyer. The perspective article concluded with open discussion among all the workshop presenters, organizers of the workshop, and final remarks from SNIP President Dr. Sulie L. Chang.

\section{Brief Reports}

\section{Cannabis, CBD, and COVID-19}

A brief report from Dr. Jag Khalsa et al. (2021) discussed the role of cannabis and its byproduct, cannabidiol (CBD), in COVID-19-associated pathogenesis. Since these substances are legal in most states for either medical or recreational purpose with easy availability, cannabis and CBD have been discussed for the treatment of COVID-19-associated mental/neurological complications and substance use disorders. However, as described in this report, there is a lack of clinical studies that support the use of cannabis or CBD for the treatment of COVID19-associated mental health and neurological complications. Thus, there is an urgent need to study the clinical role of cannabis or CBD on COVID-19-associated neurological complications. Until then, physicians/psychiatrists must caution while prescribing or recommending cannabis or CBD for treatment in COVID-19 patients.

\section{Disruption Of Blood-Brain Barrier Integrity in SARS-CoV-2 Infection}

Dr. Galie's lab has established a compliant 3D blood-brain barrier (BBB) microfluidic model. Based on the following two premises, 1) SARS-CoV-2 spike protein disrupts blood-brain barrier (BBB); 2) It is not known if angiotensin converting enzyme 2 (ACE2), the binding site for SARS-CoV-2, is involved in BBB disruption, the lab studied molecular and cellular mechanisms underlying disruption of BBB by SARS-CoV-2 spike protein using their 3D-BBB model (DeOre et al. 2021). Their studies demonstrated: 1) The expression of ACE2 was increased in response to laminar shear stress; 2) Knock down of ACE2 affected the BBB properties; 3) Spike protein triggered activation of RhoA, a key protein in regulating endothelial cytoskeleton and tight junction complex dynamics, leading to the change of $\mathrm{BBB}$ properties; 4) Inhibition of activation of RhoA by $\mathrm{C} 3$ transferase reversed the spike protein's effects. Although their spike protein studies were not able to determine if ACE2 expression is increased or decreased in SARS-CoV-2 infection, their innovative studies revealed that the engagement 
SARS-CoV-2 with ACE2 facilitates disruption of the BBB via RhoA activation.

\section{Invited Review}

\section{COVID-19 Health Disparity and Drug Abuse}

COVID-19 severely affected disadvantaged/vulnerable populations, not only with racial disparity including African Americans (AA), but also populations who used different substances or drugs. In this special issue, authors reviewed the potential cause of underlying conditions such as hypertension and diabetes and subsequent COVID-19, which disproportionally affected minorities and tobacco smokers, and their impact on renal failure (Kumar et al. 2021b). The AA population is disproportionally affected by COVID-19, in part, because they have high prevalence of underlying conditions such as obesity, diabetes, and hypertension, which are known to exacerbate COVID-19. The review also highlighted the effect of smoking on increased infection, inflammation, reduced immune response, and lung damage, leading to more severe form of COVID-19. Thus, tobacco- and various underlying conditions-induced SARS-CoV-2 pathogenesis exacerbated kidney diseases including renal failure, especially in the AA population. This review also explored the role of extracellular vesicles (EVs) in COVID-19 patients who smoke tobacco. The authors have discussed the role of EVs, which play important role in tobacco-mediated pathogenesis in infectious diseases, in COVID-19 pathogenesis and renal injurie. The review also discussed the potential role of EVs in biomarker discovery and therapeutics, which may help to develop early diagnosis and treatment of tobacco-induced renal injuries in COVID-19 AA populations.

\section{Original Research}

\section{Eugenol in COVID-19 Treatment}

Spike S1 of SARS-CoV-2 binds to ACE2 on host cells to enter the cell and initiate COVID-19. Disruption to binding between ACE2 and spike S1 could be therapeutically beneficial. Based on their ongoing studies, Dr. Pahan's lab, explored and identified Eugenol, a component of holy basil (Tulsi), capable of binding spike S1, but not ACE2, and inhibiting the interaction between spike $\mathrm{S} 1$ and ACE2 (Paidi et al. 2021). Using an AlphaScreen-based assay, only Eugenol, but not other major components of Tulsi leaf, was found to block the interaction between spike S1 and ACE2. Using an in-silico analysis and thermal shift assay, Eugenol was confirmed to associate with spike S1, but not ACE2. Entry of pseudo typed SARS-CoV-2, but not vesicular stomatitis virus, was suppressed into human ACE2 expressing HEK293 cells. Eugenol also reduced SARS-CoV-2 spike S1-induced activation of NF- $\kappa B$ and the expression of IL-6, IL- $1 \beta$ and TNF $\alpha$ in human A549 lung cells. Oral treatment with Eugenol reduced lung inflammation, decreased fever, improved heart function, and enhanced locomotor activities in SARS-CoV-2 spike S1-intoxicated mice. Taken together, the in vivo, in vitro, and in-silico studies being conducted, by selectively targeting SARS-CoV-2 spike S1, but not ACE2, Eugenol appears to be beneficial for COVID-19 treatment.

\section{Meta-analysis in COVID-19}

SARS-CoV-2 infection is known to cause complex host immune responses with cardiovascular and neurological implications. Dr. Chang's group used QIAGEN Ingenuity Pathway Analysis (IPA) and QIAGEN Knowledge Base (QKB) to conduct their meta-analysis to study the expression pattern of amyloid precursor protein (APP) upon SARS-CoV-2 infection in the brain microvascular endothelial cells (BMVECs) and during COVID-19 in progress (Camacho et al. 2021). In this study, they used 80 host response genes that are reported to be modulated in BMVECs following SARS-CoV-2 S protein binding to identify key canonical pathways and intermediate molecules mediating the regulation of APP production. The study revealed that the attachment of SARS-CoV-2 S protein may inhibit APP expression in the BMVECs. Their results shed light on the molecular mechanisms by which SARSCoV-2 infection may potentiate the incidence of stroke by inhibiting the production of APP in the BMVECs. They also analyzed molecules associated with COVID-19 identified from $\mathrm{QKB}$, which revealed six upstream regulators, TNF, IFNG, STAT1, IL1 $\beta$, IL6, and STAT3. The upstream regulators mediate the increased production of APP via intermediators, with 11 regulated by all six upstream regulators. These COVID-19 upstream regulators increased APP expression with a statistically significant Z-score of 3.705 (p-value $=0.000211)$. These findings have revealed molecular mechanisms by which COVID-19 disease may lead to long-term neurological manifestations resulting from the elevated APP expression in line with immune regulation of the host. Altogether, their study revealed two distinct scenarios which may have differential impact on APP expression. 


\section{Mitochondrial Dynamics in SARS-COV2 Spike Protein}

While $40 \%$ of COVID-19 patients develop neurological symptoms attributed to viral encephalitis, COVID long haulers develop chronic neuro-inflammation and neuronal damage, called Neuro-COVID. This study (Clough et al. 2021) from Dr. Mahajan's group tested the hypothesis that SAR-CoV-2 induces mitochondrial dysfunction and activation of the mitochondrial-dependent intrinsic apoptotic pathway, resulting in microglial and neuronal apoptosis. To test the hypothesis, they determined the effect of SARS-COV-2 on mitochondrial biogenesis and cell apoptosis in human microglia. They showed that SARS-CoV-2 spike protein increases the levels of pro-inflammatory cytokines, reactive oxygen species (ROS) production, apoptosis, and oxygen consumption rate (OCR) in microglial cells. Furthermore, non-invasive Raman spectroscopy of SARS-CoV-2-treated microglial cells showed significant difference in the composition of phospholipids, phosphatidylinositol, phosphatidylserine, phosphatidylethanolamine, and phosphatidylcholine, which account for $\sim 80 \%$ of mitochondrial membrane lipids. Overall, the study provides mechanistic insights into SARS-CoV-2-induced mitochondrial dysfunction, which underlies neuropathology associated with Neuro-COVID.

\section{Introducing the Guest Editors}

\section{Santosh Kumar, PhD}

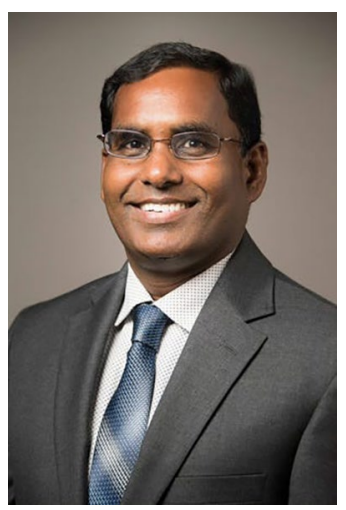

Dr. Kumar is a Professor of Pharmaceutical Sciences and an Assistant Dean in the College of Pharmacy at the University of Tennessee Health Science Center. He is also President-elect of SNIP. Dr. Kumar laboratory works on HIV, COVID-19, drug abuse, cytochromes P450, and EVs. Dr. Kumar has been investigating the role of cellular and extracellular EVs/exosomal cytochrome P450 on alcohol- and smoking-induced HIV pathogenesis and neuronal damage, which subsequently cause HIV-associated neurocognitive disorders. His group is the first one to demonstrate the expression/packaging of cytochrome P450 enzymes in plasma EVs/exosomes. His group is also involved in developing EVs as biological carriers for small drugs and other agents to treat CNS disease including HIV in the brain.

\section{Sowmya Yelamanchili, PhD}

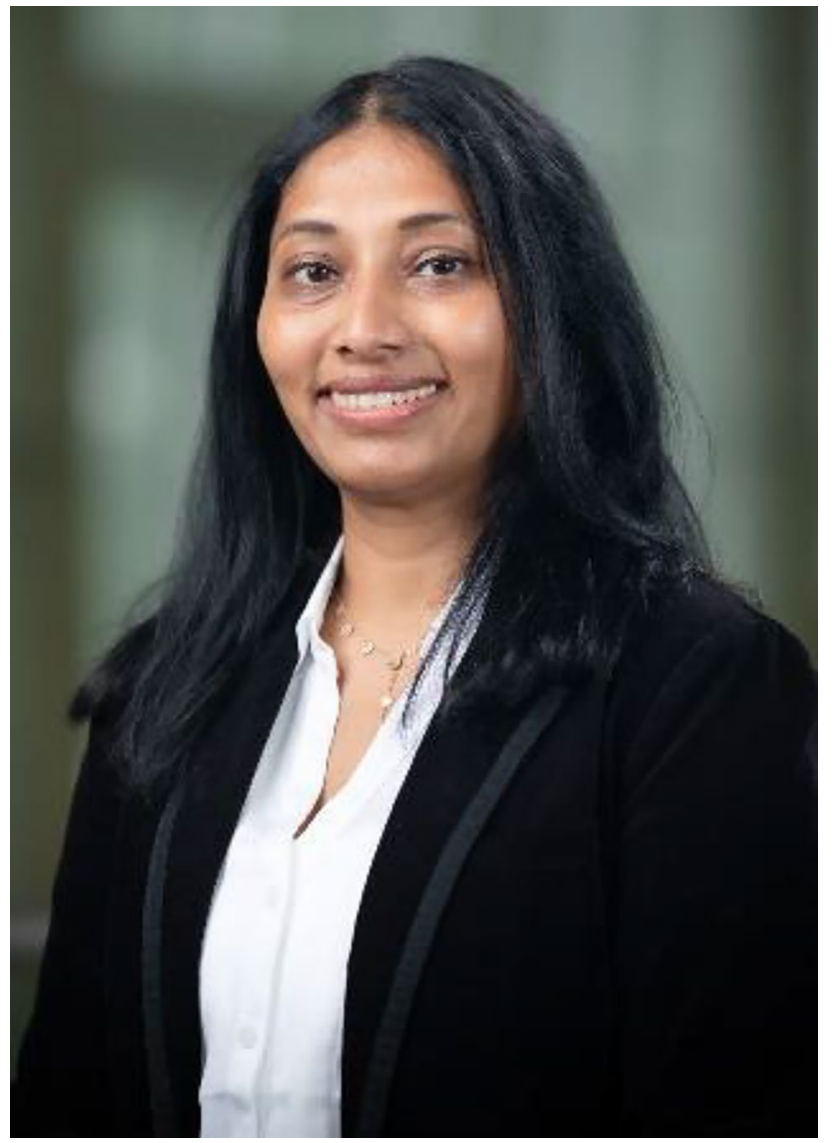

Dr. Yelamanchili is an Associate Professor in the Department of Anesthesiology at UNMC. She serves as the Interim Chair of the Diversity and Inclusion SNIP Committee. She also serves as an Executive Member for the Women's Mentoring Committee at UNMC, where she has been involved in conducting community events focusing on diversity and tackling gender-bias in science and medicine. Dr. Yelamanchili's research program focuses on understanding the role of EV and regulatory molecules such as genes, proteins, and microRNAs in the field of neuroAIDS and substance use disorder. Her laboratory has developed techniques to isolate tissue-derived EVs and she published extensively in the area of drugs of abuse. 


\section{Pankaj Seth, PhD}

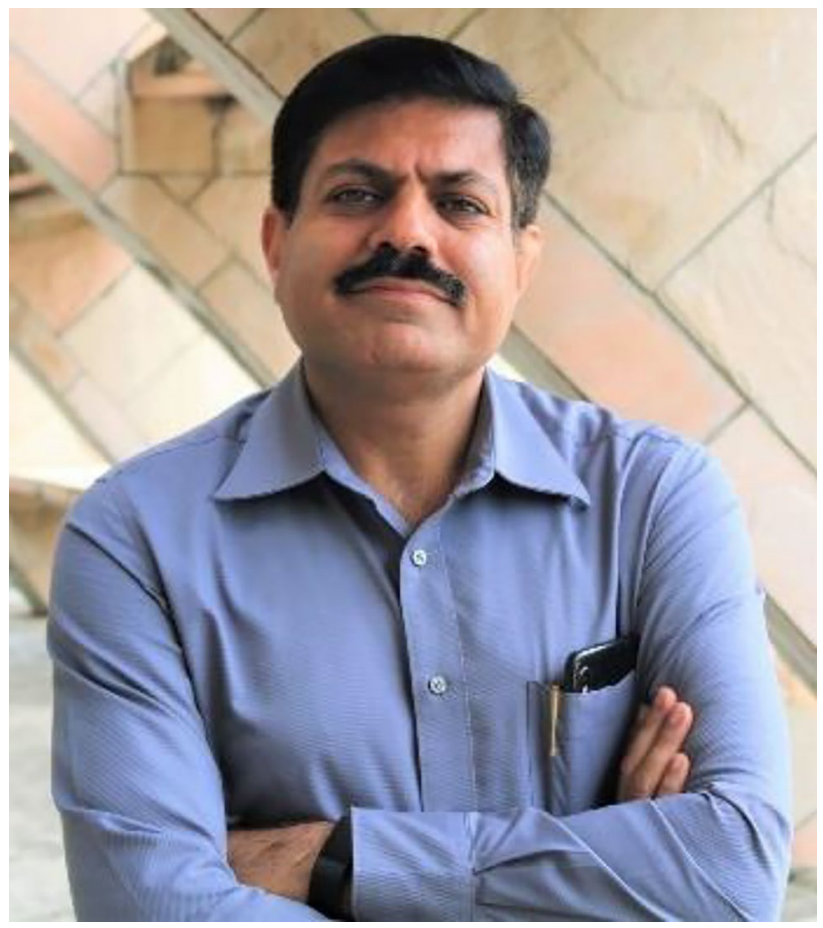

Dr. Seth is a Professor and Scientist VI at National Brain Research Centre, India. Dr. Seth's research endeavor has been of direct relevance to national health problems particularly HIV-1, drug abuse, Zika virus-mediated neuropathogenesis, and very recently effect of SARS-CoV2 on human brain. Dr. Seth was first to demonstrate that severity of HIV-1 dementia is associated with presence of dicysteine motif (C30C31) in HIV-1 Tat protein of HIV-1 subtype B. He showed that HIV-1 Tat subtype C (prevalent in India) lacks this dicysteine motif and is less neurotoxic to human neurons (Annals of Neurology 2008). Dr. Seth demonstrated HIV-1 Tat negatively impacts the properties of human neural stem cells (hNSCs) by affecting cell cycle regulator-cyclin D1 and expression profiles of genes important for proliferation and differentiation (J Neurovirology 2010). Later, he discovered the role of TRIM32, a stem cell fate determinant, in HIV-1-induced alteration of hNSCs proliferation and that TRIM32 is regulated by miR-155. He also established the role of miR-320a-VDAC1 axis in astrocyte-mediated neuronal damage in neuroAIDS. These findings were validated in human postmortem brain of AIDS patients from NIMHANS brain bank (Cell Death \& Differentiation 2016; GLIA 2017). Further, Dr. Seth demonstrated Zika virus induces microcephaly in infants by altering miRNA circuitry and pinpointed role of two miRNAs (Cell Death \& Differentiation 2018). Recently, he started research to understand why COVID19 patients exhibit neurological symptoms as brain fog. In fact, Dr. Seth had expressed this possibility in early stages of the global pandemic in April 2020 itself in a comprehensive review published by him, stating that SARS-CoV-2 is more than a respiratory virus (ACS Chem Neurosciences 2020), that is now accepted worldwide, in consonance with his vision and is being widely cited globally. With these capabilities and expertise Dr. Seth's laboratory is geared up to understand the cellular and molecular mechanisms as to how the SARS-CoV-2 affects the functioning of brain of COVID-19 patients. His research has been highlighted twice by Nature India and by the International Society of Neurovirology based in USA, electronic and print media, reflecting its impact in the field.

\section{Jean M. Bidlack, PhD}

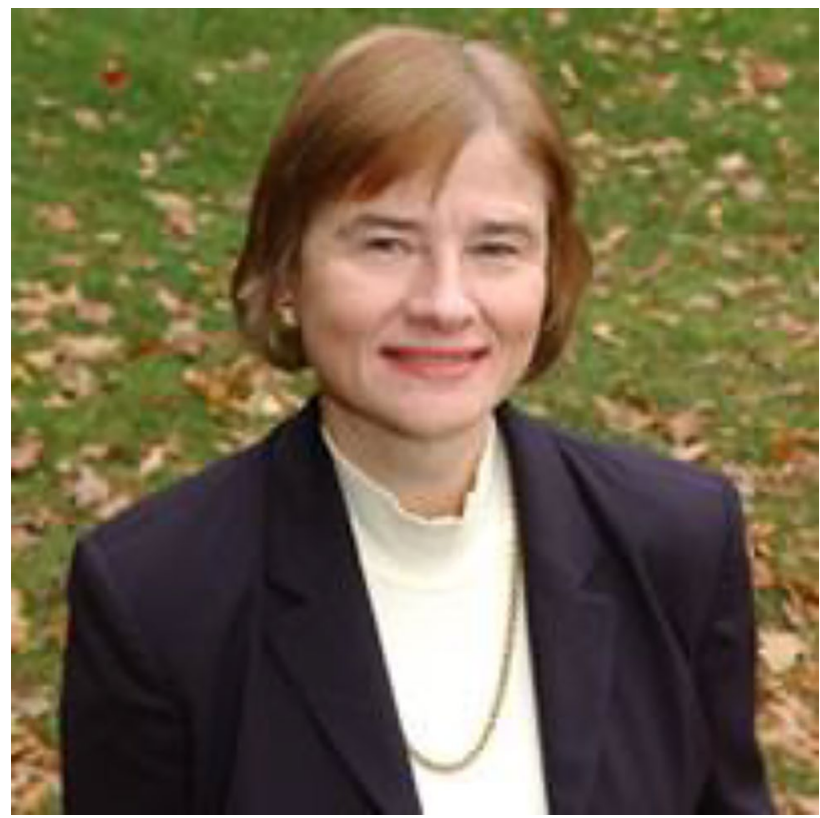

Dr. Bidlack is a Professor of Pharmacology and Physiology at the University of Rochester School of Medicine and Dentistry. Currently, she is Interim Secretary for SNIP and she is a past SNIP President. Dr. Bidlack works on studying drugs of abuse, particularly opioids. Using receptor binding, second messenger assays, and flow cytometry, Dr. Bidlack's lab was the first to identify the presence of the kappa opioid receptor on certain cells from the immune system. The Bidlack lab works on identifying novel targets for treating opioid misuse. Additional current studies use BRET technology to understand bias opioid signaling among the multiple $\mathrm{G} \alpha$ subunits and opioid receptors. 


\section{Gurudutt N. Pendyala, PhD}

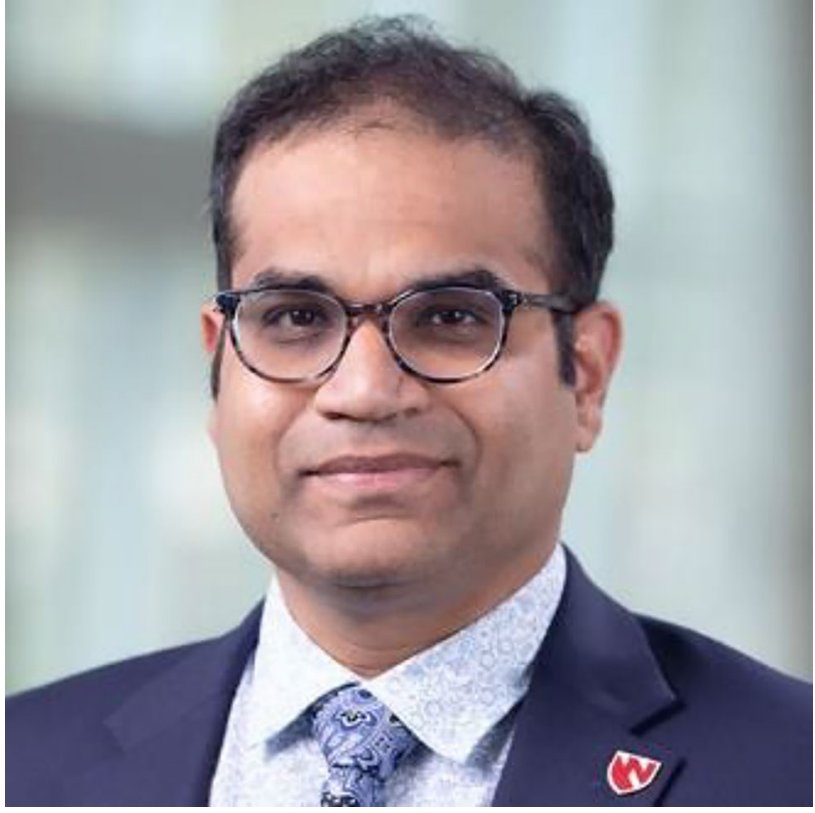

Dr. Pendyala is a Robert Lieberman Professor of Anesthesiology in the Department of Anesthesiology at UNMC. The overarching focus in his lab focuses on how drugs of abuse alone and in conjunction with HIV exacerbate CNS dysfunction with emphasis on sex differences. Another arm of his research is understanding how prescription drug abuse during and after pregnancy impacts neurodevelopment in the exposed offspring, including outcomes on biopsychosocial factors in healthcare disparities during pregnancy. A common chord connecting these arms is the application of high throughput 'omics' strategies to identify potential biomarkers in both tissue and biofluids (plasma, CSF) including decoding their associated mechanisms in vitro. Dr. Pendyala's administrative roles include serving as the Chairs of the Early Career Travel Award Committee in SNIP; Chair of the Grievance Committee in the Faculty Senate at UNMC and the founding member of the Child Health Research Institute affiliated with Children's Hospital Omaha. Recently, Dr. Pendyala was recently elected Fellow of the National Strategic Research Institute, Department of Defense, UNMC Affiliate, and also as a standing member of its Fellows Program Advisory Council.
Sanjay B. Maggirwar, MBA, PhD

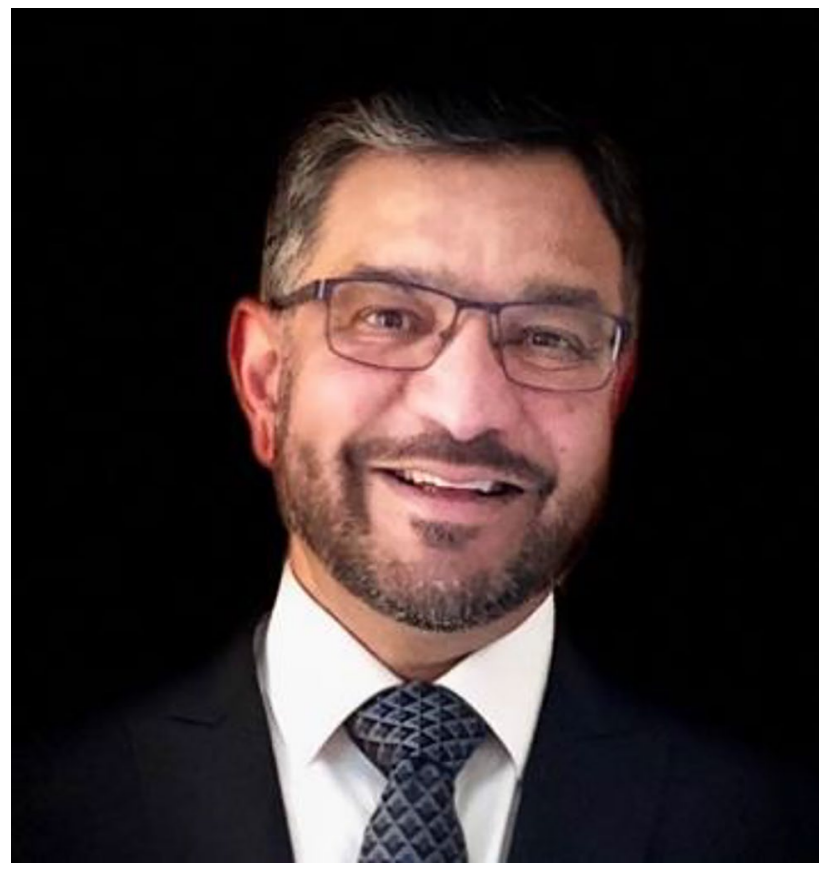

Dr. Maggirwar is a Professor and Chair of the Department of Microbiology, Immunology \& Tropical Medicine at the George Washington University School of Medicine and Health Sciences. He also plays a leading role in the Center for AIDS Research of District of Columbia as a member of its Executive Committee and Co-Director of the Basic Science Core of this organization. Dr. Maggirwar has been a HIV/AIDS researcher for over twenty-five years. In recent years, his group has studied potential relationship between HIV-infection and platelets/monocytes activation in context to neuro-HIV, aging, thrombosis and atherosclerosis. In these studies, they have also explored how the interaction of platelets and HIV-infected leukocytes mobilize the leukocytes into the CNS during acute phase of infection, thereby facilitating the seeding of brain-resident viral reservoirs. Interestingly, they also found that the $\mathrm{T}$ cells, as well as astrocytes and pericytes, latently infected by HIV exhibit defective DNA repair mechanisms, directly implicating its role in the survival of these cells. 


\section{Sulie L. Chang, PhD}

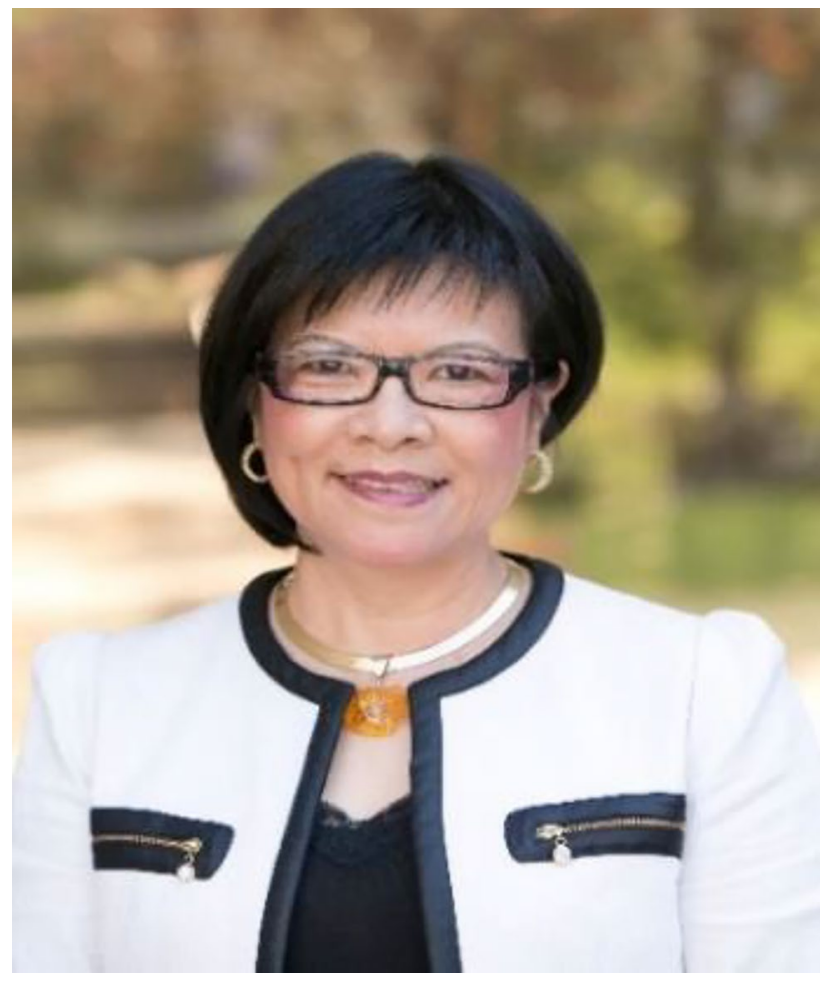

Originally, from Taiwan, Dr. Chang is Director of the Institute of NeuroImmune Pharmacology and Professor of Biological Sciences and Neuroscience at Seton Hall University. She is also an Adjunct Professor of Graduate Program in Endocrinology \& Animal Biosciences, Rutgers Graduate School. Dr. Chang is the current President of SNIP. Dr. Chang has studied the bi-directional interactions between the nervous and immune systems in health and diseases. Her research goal is to address how systemic inflammation-induced modulation of the brain-immune axis leads to behavior disorders related to addiction, cognitive impairment, and other neuronal diseases. Recently, by taking comprehensive integration of in vivo, in vitro and in silico approaches, Dr. Chang's research is to study neuroimmunology of health and diseases including spleen atrophy, alcohol abuse and drug addition, pain management, Alzheimer's disease, neuroHIV and neuroCOVID-19. Dr. Chang and her research teams have published over 145 scientific papers and book chapters with significant impact. Dr. Chang's research has been continuously funded by NIH since 1989, and she has participated in numerous NIH Study Sections.
Acknowledgements We thank the National Institute on Mental Health, MH0125670 to Dr. Santosh Kumar, and National Institute on Drug Abuse, DA046258 to Dr. Sulie L. Chang, for supporting the work. We also thank Doug Meigs, from the UNMC Department of Pharmacology and Experimental Neuroscience, for editing assistance with this guest commentary and for his overall coordination assistance on this special theme issue, "The Neuroimmune Pharmacology of SARS-CoV-2," in The Journal of Neuroimmune Pharmacology where he is Managing Editor.

\section{Declarations}

Competing Interests The authors declare no competing interests.

\section{References}

Bidlack JM, Chang SL, Fitting S, Gendelman HE, Gorantla S, Kumar S, Marcondes MCG, Meigs DD, Melendez LM, Sariyer IK, Yelamanchili S (2021) The COVID-19 pandemic: Reflections of science, person, and challenge in academic research settings. J Neuroimmune Pharmacol

Camacho RC, Alabed S, Zhou H, Chang SL (2021) Network Metaanalysis on the Changes of Amyloid Precursor Protein Expression Following SARS-CoV-2 Infection. J Neuroimmune Pharmacol. https://doi.org/10.1007/s11481-021-10012-9

Clough E, Chean KT, Inigo J, Tubbesing KE, Chandra D, Chaves L, Reynolds JL, Aalinkeel R, Schwartz SA, Khmaladze A, Mahajan SD (2021) Mitochondrial Dynamics in SARS-COV2 Spike Protein Treated Human Microglia: Implications for NeuroCOVID. J Neuroimmune Pharmacol. https://doi.org/10.1007/ s11481-021-10015-6

DeOre BJ, Tran KA, Andrews AM, Ramirez SH, Galie PA (2021) SARS-CoV-2 Spike Protein Disrupts Blood-Brain Barrier Integrity via RhoA Activation. J Neuroimmune Pharmacol. https://doi. org/10.1007/s11481-021-10029-0

Khalsa JH, Bunt G, Maggirwar SB, Kottilil S (2021) COVID-19 and Cannabidiol (CBD). J Addict Med 15:355-356. https://doi.org/ 10.1097/ADM.0000000000000771

Kumar S, Pendyala G, Yelamanchili SV, Seth P, Maggirwar S, Bidlack JM, Chang SL (2021a) Society on NeuroImmune Pharmacology COVID-19 Virtual Workshop. J Neuroimmune Pharmacol 16:519530. https://doi.org/10.1007/s11481-021-10001-y

Kumar S, Kumar P, Kodidela S, Duhart B, Cernasev A, Nookala A, Kumar A, Singh UP, Bissler J (2021b) Racial Health Disparity and COVID-19. J Neuroimmune Pharmacol. https://doi.org/10. 1007/s11481-021-10014-7

Paidi RK, Jana M, Raha S, McKay M, Sheinin M, Mishra RK, Pahan K (2021) Eugenol, a Component of Holy Basil (Tulsi) and Common Spice Clove, Inhibits the Interaction Between SARS-CoV-2 Spike S1 and ACE2 to Induce Therapeutic Responses. J Neuroimmune Pharmacol. https://doi.org/10.1007/s11481-021-10028-1

Publisher's Note Springer Nature remains neutral with regard to jurisdictional claims in published maps and institutional affiliations. 